\title{
Cooperative ITS communications architecture: the FOTsis project approach and beyond
}

\author{
Jorge Alfonso, Nuria Sánchez , José Manuel Menéndez ,Emilio Cacheiro
}

\begin{abstract}
With the continuous development in the fields of sensors, advanced data processing and communications, road transport oriented intelligent applications and services have reached a significant maturity and complexity. Cooperative ITS services, based on the idea of sharing accurate information among road entities, are currently being tested on a large scale by different initiatives. The field operational test (FOTsis) project contributes to the deployment environment with services that involve a significant number of entities out of the vehicle. This made necessary the specification of an architecture which, based on the ISO ITS station reference architecture for communications, could support the requirements of the services proposed in the project. During the project, internal implementation tests and external interoperability tests have resulted in the validation of the proposed architecture. At the same time, these tests have had as a result the awareness of areas in which the FOTsis architecture could be completed, mainly to take full advantage of all the emerging and foreseeable data sources which may be relevant in the road environment. In this study, the authors will outline an approach that, based on the current cooperative ITS architecture and the SmartCities and Internet Of Things (IoT) architectures, can provide a common convergence platform to maximise the information available for ITS purposes.
\end{abstract}

\section{Introduction}

\subsection{Background}

In the last decades, several initiatives have had a profound impact on the way road traffic and road safety are managed. Continuous development of data collection, processing and communication technologies have made available to users and traffic managers the mechanisms to increase their awareness of the traffic environment. This increased awareness of the traffic and its environment conditions is an effective tool towards improving traffic safety and efficiency, but only if the parties involved actively share their information. This is the main reason for the cooperative ITS and cooperative services concept. The European Commission has been very active in the activities towards the improvement of aspects of road safety, road mobility efficiency and transport sustainability via the promotion of ITS development. Projects like cooperative vehicle-infrastructure systems (CVIS) [1] and SAFESPOT [2] took the first steps towards using advanced communications and sensors to support the drivers in their routine tasks, steps which have lead the way to the current generation of cooperative services, exploiting the full concept of providing a complex data exchange framework between all the entities involved in the road environment.

\subsection{Cooperative ITS}

Even though the foundation of the cooperative services is arguably the underlying communications architecture [3], as the technological enabler of the whole cooperative framework, cooperative services go beyond the communications and the data exchanges between entities to consider advanced data acquisition sub-systems and processing procedures to extend the awareness of the users, and ultimately to achieve more ambitious goals towards improving road safety, traffic efficiency and the environmental sustainability in the transport applications.
Different types of cooperative services have different underlying network requirements, depending on the users and the stakeholders involved. These comprise what could be denominated the business environment of the service (Fig. 1).

\subsection{Development towards current cooperative ITS}

The effort towards the current specification of the cooperative ITS Station reference communications architecture was in principle conducted by the COMeSafety project. Initially mainly concerned with the allocation of radio spectrum for cooperative ITS systems, the project ended up as a platform for exchanging information related to all the issues related to vehicle-to-vehicle (V2V) and vehicle-to-infrastructure (V2I) communications [4]. Two of the main contributors to the initial architecture specification were the COOPERS [5] and CVIS [1] projects. The former focused on roadside based safety telematic applications, through the notification of safety-related information to the vehicles in a motorway from the road infrastructure. The CVIS project, on the other hand, focused on the technological challenges for vehicle-centred traffic management applications. The CVIS concept of vehicle-based applications was arguably the first one to face the challenge of interoperability, as it required different car manufacturers to agree on a common system specification to ensure full functionality of their applications. Other initiatives focused on more specific applications or deployment aspects of the cooperative ITS systems. SAFESPOT aimed at creating a safety margin assistant to detect in advance dangerous situations and extending the driver's awareness of its environment [2]. SEVECOM addressed the security issues in vehicle communications [6], and GeoNet assessed the possibility of network addressing based on geographic references, to complement the end-to-end approach of IP-based communications [7].

Once the basic architecture specification was established as an ETSI standard, and later as an ISO revised document, developments have focused on particular implementations of the 


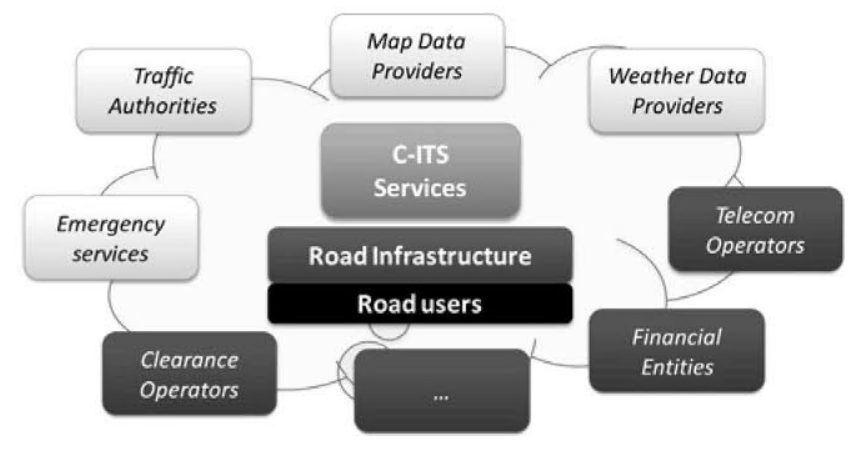

Fig. 1 Simplified cooperative services environment

architecture, and addressed missing pieces in its conception. Field operational test (FOT) projects were proposed by the EC with the purpose of evaluating the overall impact of the deployment of advanced Cooperative ITS from different points of view and considering different perspectives. Some of these were related to the impact on the users' behaviour, while others were closer to the problems of a European-wide deployment of the systems. An overall testing and analysis methodology was first established by the FESTA project, and it has since been improved with feedback from different FOT projects [8].

Different FOT projects focus on different development and deployment areas of the Cooperative Services, and each contributes to validate and advance different parts of the architecture. EuroFOT was the first large-scale FOT, and focused on the impact of several in-vehicle systems on the drivers behaviour and interactions with those systems [9]. Another relevant initiative was the TeleFOT (FOT of Aftermarket and Nomadic Devices in Vehicles) project [10]. TeleFOT was FOT project which investigated the impact of functions brought to the driver and aftermarket devices in vehicles for driver support. More recent initiatives which can be highlighted include the DriveC2X [11] and the FOTsis [12] projects. Whereas DriveC2X focuses on vehicle-centred applications as a natural continuation from the earlier efforts in cooperative ITS, FOTsis addresses specifically the integration of real-time traffic-related data from the road operators and other traffic environment entities and promotes the active participation of these entities in cooperative ITS services.

Finally, initiatives such as FOT-net data promote the sharing of information and results among FOT projects, together with the mechanisms and tools that have been employed successfully in those projects, with the objective of establishing the common ground among different FOT initiatives [13].

\subsection{FOTsis project}

The FOTsis (European FOT on safe, intelligent and sustainable road operation) project is a currently ongoing cooperative services FOT project focusing on the infrastructure side of the cooperative ITS environment, which aims to evaluate the impact on the areas of road safety, traffic efficiency and environmental sustainability of 7 close to market applications. These applications or services cover road-safety oriented services as well as road efficiency oriented services, and have been tested in four different European countries.

There are several approaches to the design of ITS services, depending on who are the final users, which are the available sources of data or which are the means to notify the final processed information to the users. The current focus of the cooperative ITS services is on the mobile entities of the road environment, therefore pushing especially concepts such as the V2V communications stack, development of on-board vehicle sensors and devices, and in general a very vehicle-oriented service design. However there are among others two factors that have to be taken into account when looking at future trends in ITS Services. One is the increasing importance of the ideas of multimodality in transport, which require a different approach to data collection than exclusively road transport environments, and the other one is the realisation that different types of resources are already deployed by the road operators and might not be fully utilised in current V2V-oriented services.

The FOTsis project takes up this second factor and develops the general concept of involving the road infrastructure in all the stages of ITS service provision. FOTsis specifically tests the road infrastructure's capability to incorporate the latest cooperative system technology at nine test sites in four countries, providing a set of seven comprehensive service entities focusing on traffic safety and mobility efficiency improvements.

- Service I Emergency management: It is an extension of the e-call concept which allows for additional sources for incident reporting and detection, and facilitates the coordination between the highway control centre and the emergency response teams. The basis of the service is the real-time tracking of mobilised resources and data exchange related to the road incident detection and resolution procedures.

- Service 2 Safety Incident management: Complementary to the previous Service 1, in this case, the main concept is to provide information about the incident to approaching road users.

- Service 3 Intelligent congestion control: In this service, collection of real-time data and the use of traffic evolution prediction algorithms make it possible to have a clear view on how the Level Of Service at a given highway stretch will progress with time. Speed recommendations are given to the drivers to minimise the impact of reduced levels of service (i.e. congestion and 'stop and go' situations).

- Service 4 Dynamic route planning: Complementary to the Service 3 , alternative routes can be recommended at selected stretches depending on the traffic circumstances and their predicted evolution. - Service 5 Special vehicle tracking: This service focuses on a specific event in the highway as it is the entry of a special vehicle. The service allows for a real-time tracking of the vehicle and the notification to other drivers of this circumstance. Information relevant for the truck driver can be transmitted in real-time as well. - Service 6 Advanced enforcement: This service notifies the driver about any conflict between the actual driving behaviour and traffic rules applicable at any given stretch (e.g. speed limits). This is done dynamically, not only considering static rules, but also any other temporary limitations set by the highway operator.

- Service 7 Infrastructure safety assessment: This service collects actual transit data for a given highway stretch, and using a driving behaviour analysis algorithm, conflictive points are identified (e.g. excessive braking sections). This allows the road operator to take the appropriate actions to eliminate or minimise the number of conflictive sections.

It is not the purpose of this paper to get into details of the services or their implementations, but just to give an overview to understand the wide range of requirements that the FOTsis architecture proposal had to comply with. Not only communications parameters were considered (e.g. bandwidth, latencies, diffusion modes etc.) but also security and external data collection needs.

\subsection{FI-PPP initiative}

At the same time, the last few years have seen the emergence of advanced ICT technologies that take the data collection and processing to new levels of availability and quantity. The FI-PPP is a European programme that aims at accelerating the development and adoption of Future Internet technologies, advance the market for smart infrastructures, and increase the effectiveness of business processes thorough the Internet [14].

The first two years of the programme focused on the development of the foundation of the technology and the capacity building and infrastructures. The main projects in this stage are FI-WARE, aiming at the specification of the common platform for FI efforts, and INFINITY [15] and XIFI [16], which focus on setting up the data infrastructures and evaluation of test infrastructure. Currently 
the effort has shifted to the development of use cases and usage areas initiatives. There are ongoing projects in areas as varied as transport and logistics, social connected TV, smart energy or e-Health, just to mention a few. The Instant Mobility project focuses on urban transportation and addresses different issues, from service operations to business models. Although Cooperative ITS elements are considered, development within Instant Mobility does not exploit aspects such as data reliability or time-criticality, and focuses on application-level data entities' exchanges. $[17,18]$

Some of the highlights of the technology trends emerged under the scope of the FI-PPP initiative are: the on-going industrialisation of IT in the form of cloud computing and open service delivery platforms and the increased network bandwidth available to the final users. Both aspects facilitate the development and provision of services in new ways, such as IT infrastructures as on-demand, pay-per-use services, real-time consumer applications in mobile data networks etc. The Future Internet vision is completed with the developments related to the Internet of Things (IoT) concept, with its vision of ubiquitously connecting intelligent devices and sensors.

\subsection{Problem definition}

The variety of entities involved in the different FOTsis services in different countries has posed a challenge, but it also offered the unique opportunity to approach interoperability issues in a global way. Throughout the project, a number of interoperability tests have been performed with other initiatives to check the proposed communications and services' architecture. Even within the FOTsis service deployments, the variety of entities involved has provided significant results in determining ways to complement different current architectural approaches to make most of the available data relevant to the road and transport environment.

However, as deployment of complex road services is becoming a closer reality, initiatives like FOTsis also help to raise awareness of aspects that could make those services more accurate, efficient and, in the end, more useful. Road traffic counters information, and in general highway operator's information, could be complemented with data coming from all the mobile devices on the highway area, and further enriched by the deployment of relatively simple data sensors.

It is widely accepted that ITS must eventually make use of all the available data sources, but to our knowledge, there has been no specific proposal on how to integrate these into the cooperative
ITS environment. Some efforts have been documented in developing very specific ITS solutions using wireless sensor network solutions [19] or addressing the service data exchanges for particular applications [20]. Notwithstanding the potential benefits of the use of the concepts of data collection and processing mechanisms in the framework of SmartCities and the (IoT), it is also necessary to realise the different approaches of the paradigms involved, and their limitations, constraints and necessary requirements before making use of data whose advantage is in quantity and ubiquity rather than quality (in terms of accuracy, reliability and dependability among others) [21].

\subsection{Objectives of the paper}

On the basis of our experience with integrating different data sources (stakeholders) in the FOTsis project, we propose in this paper a number of considerations for the integration of additional data sources into the ITS environment. We make this integration proposal not only on the conceptual knowledge of the architectures but also from our practical involvement in projects related to cooperative ITS (C-ITS) and future internet (FI), such as FOTsis, FI-WARE, INFINITY and XIFI. Some preliminary validation activities have been carried out, but it is necessary to go further into the specification of the proposed platform to perform detailed conceptual and validation tests.

\section{FOTsis communications architecture}

\subsection{Specification - FOTsis services requirements}

The specification of the FOTsis architecture starts with the analysis of the requirements of the services to be tested. It has been mentioned in the previous section the range of different requirements of the FOTsis services, including real-time data exchanges between distant mobile entities, accurate real-time traffic data collection, on-board notifications, and links with different external data providers.

\subsection{FOTsis communications architecture}

The basis of the FOTsis communications architecture is the ITS station reference architecture, as currently defined by ISO. The

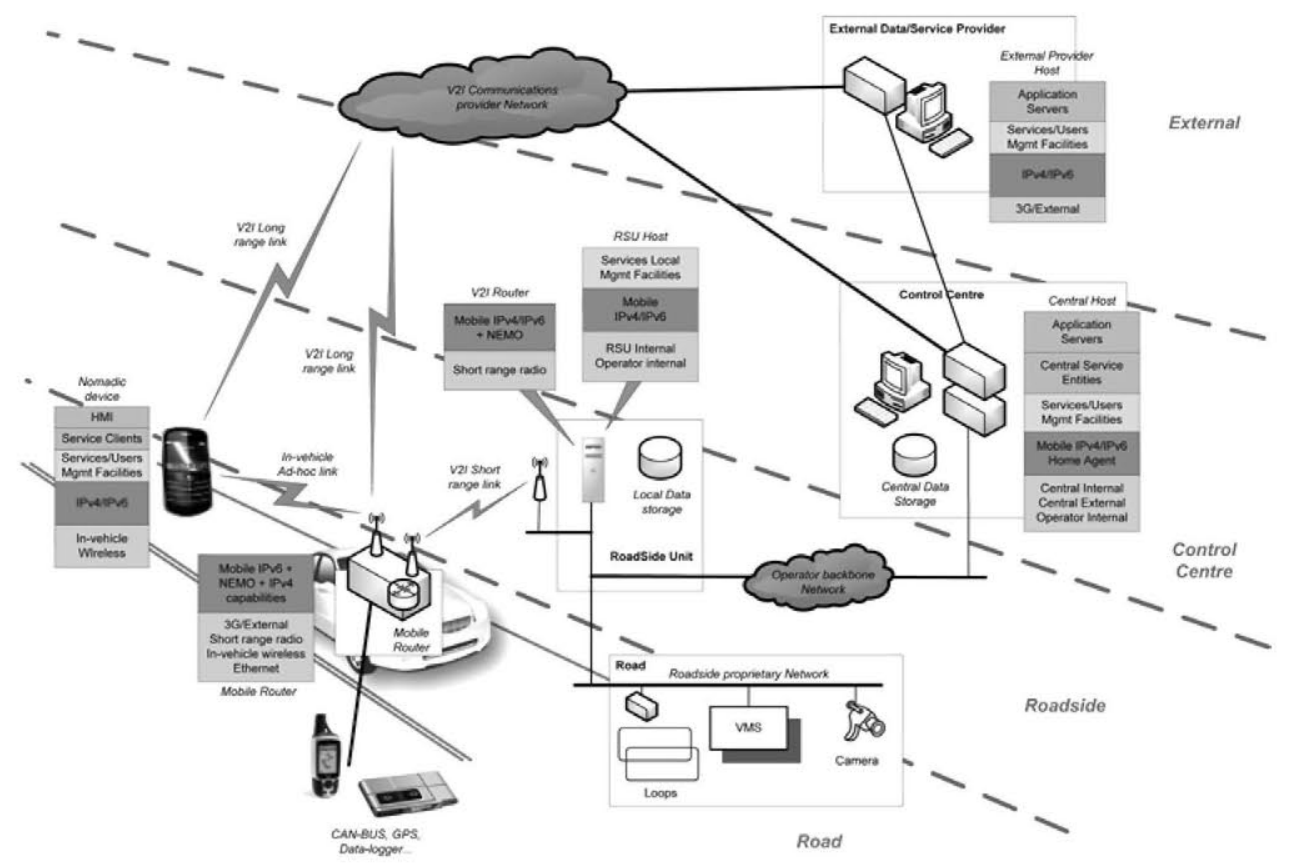

Fig. 2 FOTsis ITS Station Reference Architecture implementation proposal 
specification provides a communications framework and deployment methodology to ensure interoperability between different cooperative ITS entities [3].

The ITS-station reference architecture needs to be instantiated for each particular key element of the service, depending on its specific requirements or deployment constraints. Some of the interoperability conflicts appearing during the development of the related standards are still open (e.g. the network problem of IP-based and non-IP-based implementations of the reference architecture) and standardisation groups are working to solve them. A possible solution considers the specification of implementation profiles, for which a subset of related standards and setup parameters are chosen for specific applications.

In the case of the FOTsis communications architecture implementation, instances for nomadic devices, in-vehicle mobile link, roadside and central were specified. Details of the instances include the selection of $\mathrm{WiFi} / 802.11 \mathrm{p} / 3 \mathrm{G}$ as access technologies, and the use of CAM, DENM messages, together with two new ID tracking and geographically-based message dissemination facilities. However, the most important aspect of the FOTsis architecture is that it is completely IP-based. The use of IPv4 and IPv6 facilitates the integration of external entities in the services, and provides the mechanisms for simple mobility, interoperability across different sectors, security, and above all, scalability possibilities [22]. Fig. 2 shows the overall FOTsis architecture proposal.

\subsection{Validation of the FOTsis communications architecture}

Validation activities for the FOTsis communications architecture include the architecture pilot tests conducted at different test-sites. All of the tests were under controlled conditions, but involved a complete communications setup following the FOTsis architecture proposal. Whereas early validation activities focused on the communications network, and particularly the use of road-oriented $802.11 \mathrm{p}$ technologies, subsequent tests have addressed the performance and capabilities of the whole service chain. Data collection and transmission to the application server, normally located remotely, via the roadside units and the highway network, storage for service operation or results analysis, as well as data processing for real-time service provision have all been part of the more recent tests. Fig. 3 shows the overall view of the implementation in one of the tests carried out in one of the Portuguese test-sites. Other tests performed in Spain, for example, had a different configuration to facilitate interoperability checks and account for potential differences in implementation of the service in real life (different manufacturers for different elements, different mobility setups etc.).

\subsection{Future internet architectural approach}

One of the goals of the FI-WARE project is the specification of the core platform for the Future Internet. The Future Internet (FI) platform is open, based on elements called Generic Enablers, which offer reusable and commonly shared functions for different target areas across various sectors.

The future internet architecture must also be instantiated into a particular implementation by selecting and integrating a number of generic enablers (GE). The specific resulting implementation is defined by a number of characteristics tailored to the requirements of the application in terms of quality specifications and necessary resources (Fig. 4).

\section{Integrating the FOTsis cooperative ITS communications architecture and FI data sources}

\subsection{Integration of additional data sources in the cooperative ITS environment}

Developments carried out for the specification of the FOTsis architecture have resulted in a detailed knowledge of part of the Cooperative ITS (C-ITS) environment and its relevant actors, as pointed out in Section 1.

One of the main characteristics of the ITS Station reference architecture is its design as a secure bounded environment. Some of the C-ITS services are related with safety, and all the data exchanged and processed in the system must meet critical requirements regarding accuracy, reliability and integrity. In addition, many of the services developed may have tight time constraints. A typical C-ITS application is the incident ahead warning, which relies on timely distribution of information to the vehicles around the incident to prevent secondary incidents.

On the other hand, the strength of the Future Internet approach is based on the ideas of ubiquitous connection of devices and sensors and extended connectivity to the users, somehow creating a virtual pool of data and services that can be accessed in different ways. The key aspect when combining these two approaches lies in the realisation of the limitations and constraints that each imposes on the data exchanges at different levels, and the importance of the management in handling these limitations. The fact that both architectures have identified separated management and security elements facilitates the preliminary identification of elements related to the management and control of data flows and processing, and allows the conceptual design of a combined architecture.

It is important to consider that the overall design of the FI architecture facilitates integration of different systems and networks. Additionally, C-ITS applications and the corresponding underlying reference architecture implementations often impose tight requirements on data and processes. These two facts have as a consequence that the proposed approach to the integration of C-ITS systems and FI structures should be based on the idea that C-ITS can be considered as a particular use case of FI implementation. This is one of the aspects that are being included in the preliminary validation tests being currently carried out.

As a starting point to the integration concept, we establish different deployment scenarios that can be used to illustrate the

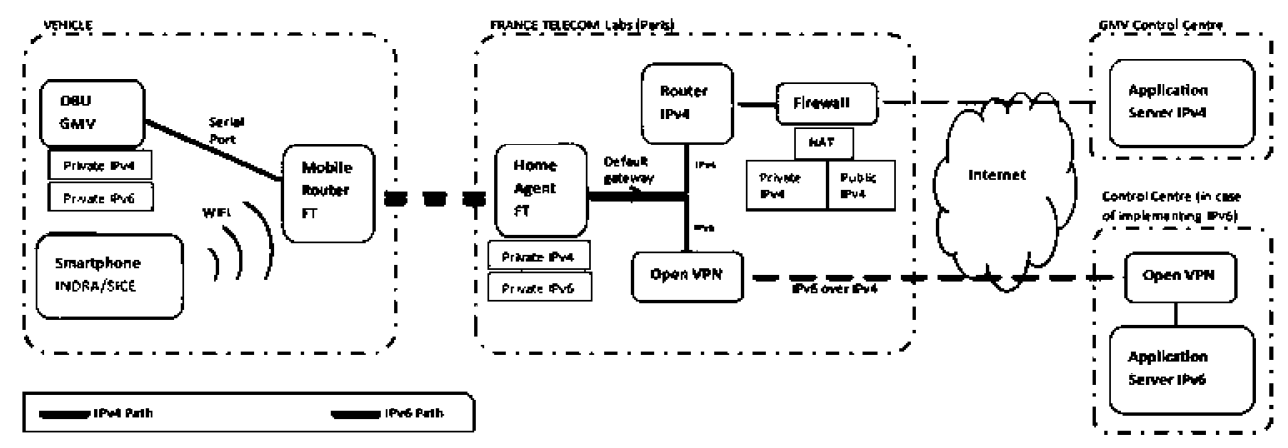

Fig. 3 FOTsis architecture pilot test implementation example 


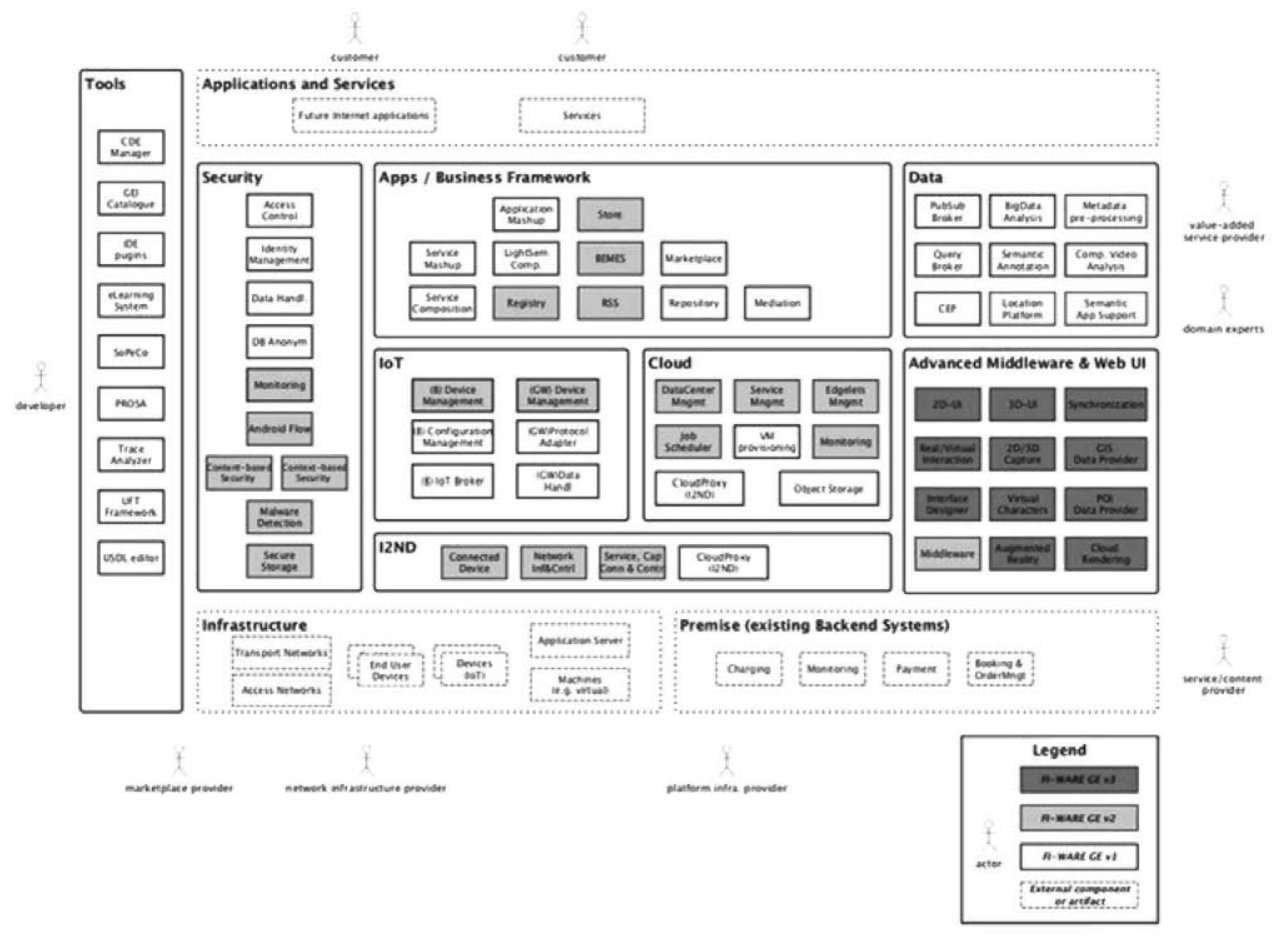

Fig. 4 Diagram of the FI-WARE common platform (Source: FI-WARE)

integration concept described in this paper. For the purpose of simplicity, we simplified the functionalities of the services, and we considered basically only the data collection for applications.

With these constraints, we specify two deployment scenarios:

- A C-ITS-based vehicle host wants to make use of available FI resources. A possible application is a POI information dissemination hot-spot for nearby vehicles or a public street parking availability service based on road sensors deployed specifically to monitor and advertise street parking places availability. This framework is obviously more useful when considering the road or traffic manager point of view, and results in an increased awareness of the traffic-related environment.

- A FI-based host wants to make use of available C-ITS resources. In this case we may have a traffic manager that, besides its own detection devices, may want access to all the advanced C-ITS-based data sources on the road, and additionally, to the available FI-based data sources, fixed or mobile.



Fig. 5 Interface to networks and devices (I2ND) global architecture diagram (Source: FI-WARE)
The service in this case can be the hazard warning to the users (which is one of the services being tested in FOTsis). And while this service is already considered as a cooperative service, the integrated proposal in this paper makes it possible to extend the awareness of the road operator of the hazards to be notified. The C-ITS service is essentially a geographic-based advertisement service for approaching drivers. The involvement of the road operator and the integration of additional data sources to the service adds a greater awareness of potential hazards. Wireless sensor networks could now be seamlessly integrated in the data flow of the operator.

In the FOTsis project, weather-related information and warnings are already integrated in the services. For this purpose, an advanced road weather model has been designed which monitors readings from the available weather stations nearby the highway and makes accurate predictions on weather evolution. Weather data and warnings are sent through IP-based web-services. Other environmental variables, such as the road state, could be easily added. This flexibility and ease of integration are a very significant advantage of the use of IP-based communications in the FOTsis architecture, and also provides an edge over other (non-IP) Cooperative ITS implementations when considering integration with FI-based devices.

\subsection{C-ITS integration in the FI framework}

We focused on the I2ND (Interface To Network devices) block of the FI architecture. It defines an enabler space for providing other GEs to run an open and standardised network infrastructure. In other words, this is the block that is closer to the management of the network resources and is the one that will be necessary to consider for integration with the C-ITS management tools (Fig. 5).

The I2ND block in turn includes four generic enablers, with specific functionalities and interfaces according to their underlying technologies

- CDI. Connected device interface, towards end-user terminals (nomadic devices, tablets, set top boxes etc.)

- CE. Cloud edge, towards cloud proxy gateways.

- NetIC. Network information and control, towards open networks. 
- S3C. Service Capability, Connectivity and Control, towards underlying networks, which in principle should follow the EPC (Evolved Packet Core) 3GPP specification.

The $\mathrm{S} 3 \mathrm{C}$ generic enabler is intended to be the central control and management entity. The fact that FI has selected EPC as its baseline structure can be seen as an advantage for integration, because EPC was designed as an IP-based connectivity control platform, and maintains previous $3 \mathrm{GPP}$ platforms capabilities, most notably QoS policies management, mobility, charging, security etc.

However, the FI approach to network imposes a number of constraints to how resources have to be managed and controlled. At the current state of development, it seems necessary that Open Networks and EPC-enabled networks are considered to be a part of the FI environment.

\subsection{Integrated platform concept overview}

We have mentioned how the FI architecture aims at maximising the availability of resources for the services at any given moment. This idea has led to an architectural design based on the development of data collection and processing paradigms and the elements necessary to handle the management and control of those data paradigms. The two main technologies integrated in the FI platform are the cloud and the IoT approaches. In the same way, we can consider the possible issues of integrating the C-ITS paradigm into the FI overall structure.

One of the main differences between C-ITS and the current implemented ideas of FI is that from an implementation point of view, the C-ITS architecture is a much tighter and vertical effort. Since the requirements of the security applications are well-known, the whole structure can be more focused, and the resources more controlled at any and every level. This is particularly true of the lower levels, such as access, network and transport, in relation to which the reference architecture is complete, and missing some complementary elements to insure interoperability between different implementations of the architecture. Above the transport level, however, the specification is still not complete. While some applications are being developed and standardised, it is still not clear which are the mechanisms by means of which applications can secure underlying resources to meet their requirements. A number of facilities tools have been defined to address certain common functionalities such as encryption, compression or more data related, such as positioning exchanges or time information.

On the other hand, FI applications are in their current specifications, more focused on the data exchanges and the higher layer aspects of the deployments. There have not been many initiatives for area developments, which means only a few communication and network profiles have been already specified. Some of these are related to health applications (FI-STAR, [23]), smart cities and energy (FINESCE, [24]), leaving essentially the mentioned FI-WARE, XIFI and INFINITY as the infrastructure and architecture related efforts in FI.

When facing the issue of how to integrate a C-ITS entity in the FI environment, we need to look again at the specific characteristics of the C-ITS entities: secure and bounded data exchangers, with QoS (e.g. time delays and data production frequency) built from design, and currently being under further development in the shape of flow management facilities.

The integration proposal we make is based in the exploitation of these characteristics and their implementation in the I2ND block of the FI architecture. The key aspects of this integration would be the addition of an EPC adaptation layer to the C-ITS elements and the incorporation of the C-ITS flow management and application interfaces into the functionalities of the FI S3C GE. The overall concept can be seen in Fig. 6.

\subsection{First step towards integration - an advanced application data flow management}

As stated in the previous section, the key aspect of the integrated architecture must be the development of a complete management entity designed focused on the applications and end-user services, and their specific data requirements. This management entity would integrate the Cooperative ITS approach to management, in which applications or services dictate the resources they would need (e.g. in terms of bandwidth and security), while still

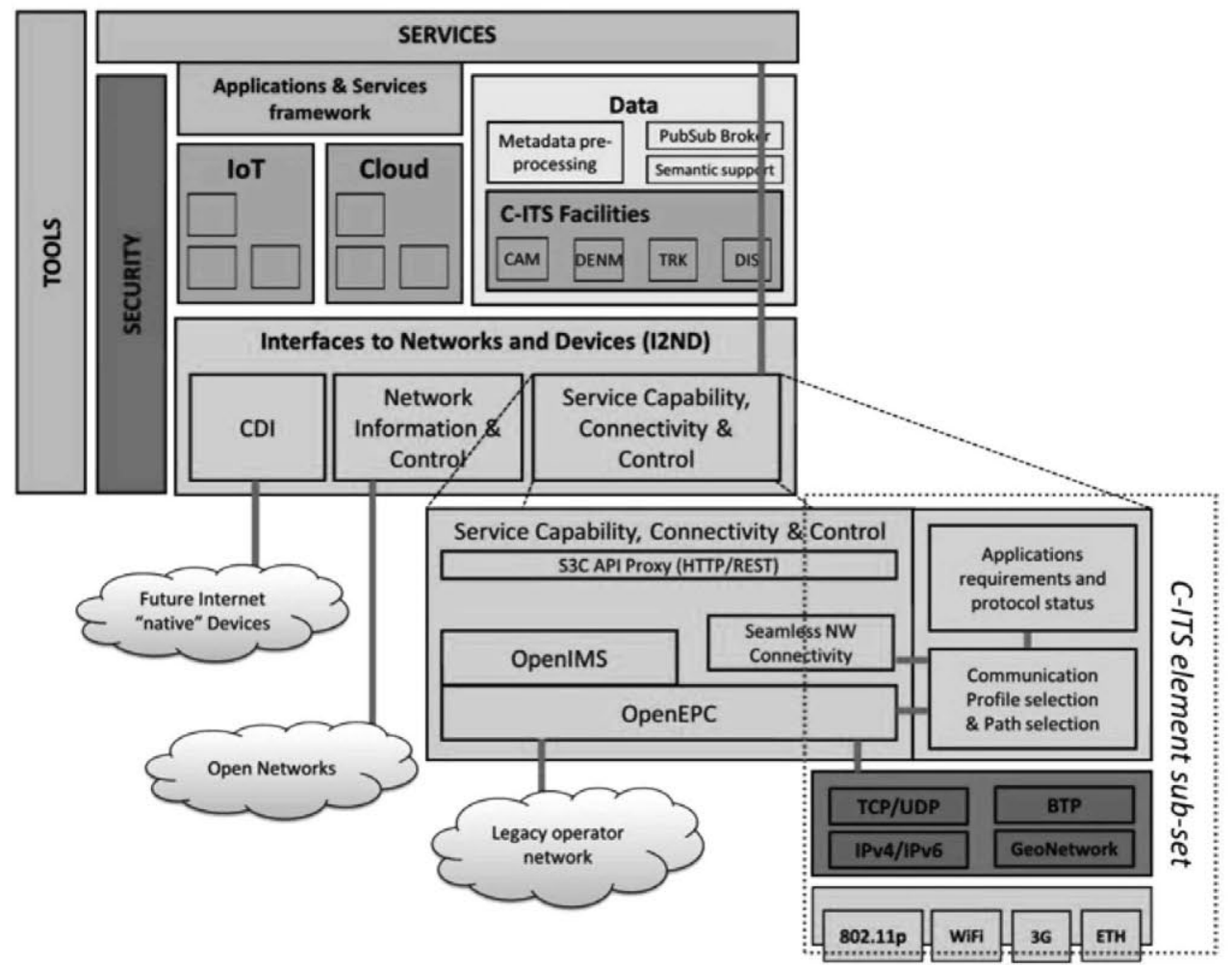

Fig. 6 FOTsis Cooperative ITS and Future Internet convergence architecture 


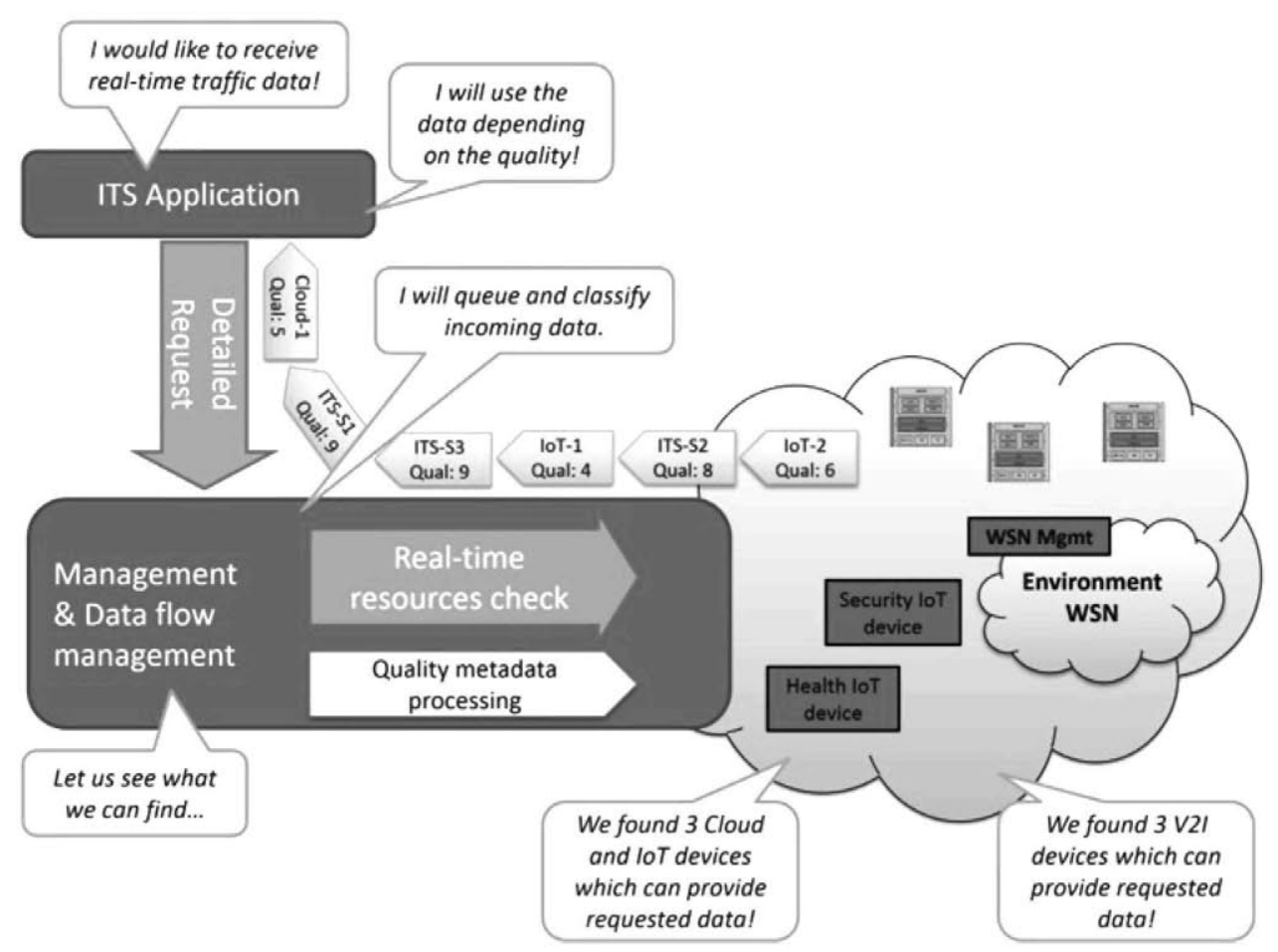

Fig. 7 Integrated quality-based data flow management

complying with the more complex future internet approach. The FI management is more distributed in different elements, but in the end, an application has a maximum specified awareness of the environment resources through a number of generic enablers I2ND 'network information and control' GE, CloudProxy, IoT device management etc... - hence requirements can equally be met by the corresponding securing of resources. One of the goals of the integration is to define the mechanisms by which the management entity acquires complete awareness of resources and can handle both C-ITS and FI elements seamlessly for the applications, and secure dynamically the resources they need.

There are at least two interfaces in the $\mathrm{S} 3 \mathrm{C}$ generic enabler that allow the management of QoS parameters:

- Seamless network connectivity, which enables access of the rule sets from external services.

- OpenEPC, which allows the services to transmit directly their requirements towards the core network. It provides the means for applications to reserve resources and thus realise the required QoS characteristics. In addition, it provides the means for the applications to access network information and specific subscribers data.

The effort is focused now on integrating these interfaces with the intended flow management functionalities and quality control of the ITS Station instances. Once this is achieved, ITS Station instances can be seamlessly integrated into the FI environment, and will be able to provide the FI applications with secure, high reliability traffic-related data. We can think of very accurate vehicle warnings, traffic light status and control etc.

We have seen how we propose the concept of advanced data flow management as the key to handle different data pieces. It is our understanding that the main issue for applications is to know exactly how accurate and reliable the information is, when received from different unknown sources. For example, for a traffic management application, data about traffic volume in a road stretch could come from different sources: traffic sensors on the road, V2I messages from C-ITS devices onboard the vehicles or information reported from drivers' mobile applications, among others. However, it is not possible to consider all this information to have the same quality. The specification of the elements necessary to quantify the quality parameters of the information can imply different aspects: from the definition of quality metadata, to the specification of the tools that can support the processing and metadata exchanges, modifications in the FI management to integrate C-ITS aspects and the quality metadata management etc.

A Fig. 7 shows in a simplified way the main interactions of the advanced data flow concept and its links with different elements of the current architectures. We can see also the importance of having a common implementation of quality parameters. Here we have just illustrated a single quality parameter, but the idea is to have a set of quality parameters which can be used by applications, tools, management elements, network and security elements in implementations of the architecture to properly handle the information being exchanged in the environment. There have been already some efforts to specify a set of quality parameters for ITS applications, as can be seen in [25].

\section{Discussion and conclusions}

The current cooperative ITS scenario presents several issues and challenges. Some of them are related to the completion of the tools to make different implementations of the ITS-station reference architecture fully interoperable. Others are related to the fact that it is possible that a significant amount of data relevant for ITS purposes is not currently used by ITS services.

In this paper, we introduced a possible approach to an integration of cooperative ITS services architecture and future internet structure aiming at providing a solution that can maximise the data available about the road environment. This approach is based on the idea that C-ITS could be considered a particular case of a FI implementation. The goal is therefore to identify the elements that are necessary to address for the integrated management to make use of both the virtualised and distributed resources of FI as well as the tightly controlled C-ITS resources on demand and seamlessly for the applications.

We introduce what could be one of the steps towards the final goal, the implementation of a quality metadata management and control sub-system that would be one of the bases of the combined architecture and handle the procedures of quality measurement and assignment, security aspects and advanced flow control. All the information available to the applications is therefore measured by 
the same standards in relation to quality aspects, independently from the source or the processes undergone in the way, and can thus have more resources available for their operations. In an increasingly connected world, this is a way to ensure that ITS services have the maximum awareness about the environment and can achieve more successfully the goals of road safety, mobility efficiency and environmental sustainability.

Although some preliminary tests have been carried out to validate some of the main integration concepts presented in this proposal, more detailed tests are necessary to address the more complex aspects of the combined architecture. Involvement in major projects like FOTsis and FI-WARE is proving critical to facilitate partial validations of the concept presented.

In these preliminary steps, we have focused on the data collection stage of the applications, and proposed a way to integrate data from different Cooperative ITS and FI sources seamlessly into the FI environment. We already pointed out that processing and presentation of service data to the users could be another interesting line of work, and is one that will probably be taken into account in the near future. In particular, making use of distributed data and processing resources could open the way to significant changes in the way ITS services are designed.

Depending on the road services to be integrated, there are other challenges for the specification of this common platform. Electronic fee collection and in general in-vehicle payment facilities developments are still relatively isolated from C-ITS and FI, but are a key element in attaining the objective of a single service platform for road services.

\section{Acknowledgments}

This work has been funded partially under the FOTsis project which is partly financed by the European Commision in the context of the 7th Framework Programme (FP7) for Research and Technological Development (grant agreement no. 270447). In addition, this work was partially supported by the Centre for Industrial Technological
Development (CDTI) under Project INNPRONTA Ciudad2020 IPT-20111006.

\section{References}

CVIS: 2010. Available at www.cvisproject.org, accessed August 2014

2 SAFESPOT: 2010. Available at http://www.safespot-eu.org/, accessed August 2014

3 ISO 21217:2014: 'Intelligent transport systems - communications access for land mobiles (CALM) - architecture', 2014

4 COMeSafety: 2009. Available at www.comesafety.org, accessed August 2014

5 COOPERS: 2010. Available at http://www.coopers-ip.eu/index.php?id-project, accessed August 2014

6 Kroh, R., Kung, A., Kargl, F.: 'SEVECOM D1.1 VANETS security requirements final version', 2006

7 Ernst, T.: 'GeoNet D1.2 final GeoNet architecture design', 2010

8 FOT-Net Consortium: 'FESTA handbook version 5', 2011

9 EuroFOT: 2012. Available at www eurofot-ip eu accessed December 2014

10 TeleFOT: 2012. Available at http://www.telefot.eu/, accessed September 2013

11 DriveC2X: 2014. Available at http://www.drive-c2x.eu/project, accessed August 2014

12 FOTsis: 2013. Available at www.fotsis.com, accessed March 2014

13 FOT-Net Data. Available at www.fot-net.eu, accessed December 2014

14 FUTURE INTERNET PPP Home: 2013. Available at http://www.fi-ppp.eu/, accessed April 2014

15 The INFINITY Project: 2013. Available at http://www.fi-infinity.eu/portal, accessed April 2014

16 XIFI: 2013. Available at https://fi-xifi.eu/home.html, accessed April 2014

17 Nagellen, N.: 'Instant mobility D7.9: Instant mobility standardization and regulation recommendations - final version', 2013

18 Peña, C., Dautelle, J.M., Zeddini, B., et al.: 'Instant mobility D2.5: Recommended infrastructures for pilots'. Instant Mobility, 2012

19 Barone, R.E., Tullio, G., Siniscalchi, S.M., Morgano, M.A., Tesoriere, G.: 'Architecture for parking management in smart cities', IET Intell. Transp. Syst., 2014, 8, (5), pp. 445-452

20 Pagano, P., Petracca, M., Alessandrelli, D., Salvadori, C.: 'Is ICT mature for an EU-wide intelligent transport system?', IET Intell. Transp. Syst., 2013, 7, (1), pp. 151-159

21 FI-WARE: 2013. Available at http://forge.fi-ware.org/plugins/mediawiki/wiki/ fiware/index.php/Main_Page, accessed August 2014

22 Alfonso, J., et al.: 'FOTsis deliverable D3.7 FOTsis trials architecture', 2013

23 FI-STAR: 2013. Available at www.fi-star.eu/fi-star.html, accessed August 2014

24 FINESCE: 2013. Available at www.finesce.eu, accessed August 2014

25 ISO/TR 21707:2008: 'Intelligent transport systems - integrated transport information, management and control - data quality in ITS systems', 2008 